\title{
Urologische Notfälle: Klinische Präsentation, Diagnostik und Therapie
}

\author{
Peter J. Olbert, Axel Hegele, Martin Ludwig
}

\section{Übersicht}

Einleitung

Akutes Skrotum

Erweiterte Differenzialdiagnosen

Klinische Untersuchung

\section{Einleitung}

Ein nicht unerheblicher Anteil der notfallmäßigen Zuweisungen in eine Klinikambulanz betrifft unmittelbar das Teilgebiet der Urologie. Dieser Übersichtsbeitrag soll einen kurz gefassten Überblick über die häufigsten und typischen urologischen Notfallszenarien mit ihren Differenzialdiagnosen, der angemessenen Diagnostik und den notwendigen therapeutischen Schritten geben.

Da eine spezifische Therapie häufig erst in einer Facharztpraxis oder Klinik möglich ist, gilt auch hier, dass das Daran-Denken bei bestimmten klinischen Erscheinungsbildern häufig entscheidend ist. Dieser Beitrag wurde für die bessere Übersichtlichkeit in die 3 klinischen Symptomkomplexe

- akutes Skrotum

- Flankenschmerz

- Harnverhalt/Makrohämaturie

unterteilt. Um den vorgegebenen Rahmen nicht zu sprengen, wurde bewusst auf eine Abhandlung der urologischen Traumatologie verzichtet. Da Verletzungen der Urogenitalorgane häufig Begleitverletzungen im Rahmen komplexerer traumatologischer Szenarien sind, erscheint eine Aufarbeitung zum Beispiel im Rahmen der Polytrauma- oder Beckenfrakturversorgung sinnvoller.

\section{Akutes Skrotum}

Als „akutes Skrotum“ werden akut einsetzende Schmerzen im Skrotalbereich bezeichnet, die oft mit begleitenden Symptomen wie Skrotalschwellung, Rötung oder Überwärmung einhergehen. Verschiedene Erkrankungen können pathogenetisch auslösend sein, die auch in der Notfallsituation unterschiedliche Therapieansätze erfordern. Typisch für das akute Skrotum ist die rasche Mitbeteiligung initial nicht beteiligter benachbarter skrotaler Strukturen, die die Differenzialdiagnose erheblich erschweren kann.

Die folgenden Erkrankungen sind beim akuten Skrotum zu berücksichtigen:

- Samenstrangtorsion (synonym Hodentorsion)

- Hydatidentorsion

- akute Epididymitis

- Orchitis

- Hodentrauma

\section{Samenstrangtorsion}

Unter einer Samenstrangtorsion wird eine axiale Drehung des Hodens um den Samenstrang bezeichnet. Ursächlich liegt wahrscheinlich eine Hypermobilität durch Cremasterfaserkontraktion vor. Es werden $2 \mathrm{Al}-$ tersgipfel bei Kleinkindern und jugendlichen Männern 
beschrieben, die Inzidenz soll etwa 25/100000 jugendlicher Männer betragen [1]. Anatomisch lassen sich die extravaginale, intravaginale und mesorchiale Form der Torsion unterscheiden, die Differenzierung ist allerdings für Akutsituation und Therapie irrelevant.

\section{Hydatidentorsion}

Hydatiden werden in definierten Lokalisationen beschrieben: als Morgagni-Hydatiden am oberen Pol des Hodens oder im Bereich des Nebenhodenkopfs, als Paradidymis im Bereich des benachbarten Samenstrangs oder eines Vas aberrans. Torsionen dieser anatomischen Strukturen finden sich in älteren Studien bei etwa einem Drittel der Hodenfreilegungen wegen vermuteter Samenstrangtorsion, da Diagnostik und klinische Symptomatik oft keine Unterscheidungsmöglichkeit bieten.

\section{Akute Epididymitis}

Die Epididymitis trägt zu ca. 95\% aller Fälle mit akutem Skrotum bei [1]. Sie entsteht entweder bakteriellaszendierend nach vorangegangener Urethritis (bei jungen, sexuell aktiven Männern), oder kanalikulärurinogen bei bestehender Harnwegsinfektion (bei älteren Männern mit subvesikaler Obstruktion). Dementsprechend unterscheidet sich das Erregerspektrum: Während bei jungen Männern insbesondere sexuell übertragbare Keime wie Chlamydia trachomatis und Neisseria gonorrhoeae pathogenetisch im Vordergrund stehen, sind bei älteren Männern Enterobacteriaceae, insbesondere Escherichia coli, von Bedeutung. Im Rahmen einer Urogenitaltuberkulose kann eine Epididymitis durch Mykobakterien auftreten [2].

Nicht infektiöse Ursachen sind von eher untergeordneter Bedeutung [3]:

- urinogen-chemisch: zum Beispiel nach transurethraler Resektion der Prostata

- bei Allgemeinerkrankungen: zum Beispiel bei Morbus Behçet

- nach Vasektomie: „blow out“-Verletzung des Nebenhodengangs

- medikamenteninduziert: zum Beispiel bei Amiodaron

Trotz intensiver Diagnostik bleiben auch heute noch 40-50\% der Epididymitiden ätiopathogenetisch unklar [4].
Ätiopathogenese der Epididymitis = Harnwegsinfekt oder Urethritis oder nichtinfektiös oder idiopathisch.

\section{Orchitis}

Im Gegensatz zur Epididymoorchitis, bei der die Entzündung vom Nebenhoden auf den Hoden übergreift, handelt es sich bei der seltenen primären Orchitis um eine isolierte Entzündung des Hodens, wobei der hämatogene Infektionsweg im Vordergrund steht. Neben einer Reihe bakterieller und viraler Ursachen tritt die Erkrankung im klinischen Alltag aber zumeist als eine Begleitorchitis bei postpubertärer Mumpserkrankung durch Paramyxoviren auf. Sie folgt der Parotitis mit einer Latenzzeit von 3-10 Tagen, tritt bei 20-30\% aller postpubertal erkrankten Männer auf, in 30\% bilateral [3]. Die Gefahr liegt in der postentzündlichen Entwicklung einer Hodenatrophie 2-3 Monate nach der Infektion.

\section{Hodentrauma}

Es wird eine penetrierende Verletzung vom stumpfen Hodentrauma unterschieden. Beim penetrierenden Trauma handelt es sich oft um ein komplexes Verletzungsmuster nach Schuss-, Biss- oder Pfählungsverletzungen. Beim stumpfen Hodentrauma stehen Hodenruptur, Hodendislokation oder lediglich ein Skrotalhämatom im Vordergrund.

\section{Erweiterte Differenzialdiagnosen}

Hodentumoren, Varikozelen oder akut einsetzende Hydrozele können in Einzelfällen eine akute Schmerzsymptomatik bieten, spielen aber in der engeren Differenzialdiagnose des akuten Skrotums nur eine untergeordnete Rolle.

Als erweiterte Differenzialdiagnosen sind weiterhin Erkrankungen zu nennen, die zwar primär außerhalb des Skrotums lokalisiert sind, aber eine skrotale Schmerzausstrahlung oder eine skrotale Mitbeteiligung aufweisen können.

Bei der inkarzerierten Leistenhernie folgt dem akuten Scrotum ein akutes Abdomen, bei skrotaler Lage lassen sich auskultatorisch oft Darmgeräusche, sonografisch eine intraskrotale Peristaltik nachweisen. 
Harnleiterkoliken können je nach Lokalisation des Abflusshindernisses in Hoden oder Glans penis ausstrahlen und eine primär skrotale Schmerzgenese imitieren.

Auch bei der akuten Appendizitis ist je nach Lage der Appendix ein fortgeleiteter Schmerz auch in das Skrotum bekannt.

Bei skrotalen Schmerzen auch extraskrotale Ursachen berücksichtigen.

\section{Klinische Untersuchung}

In der Praxis stellt die Differenzialdiagnose zwischen Hodentorsion und akuter Epididymitis die größte Herausforderung dar. Anamnese und Lokalbefund sind dabei richtungsweisend, können aber letztendlich die korrekte Diagnose nicht beweisen.

Klinisch am bedeutendsten ist die Differenzialdiagnose zwischen Hodentorsion und akuter Epididymitis.

Anamnese und Lokalbefund sind richtungsweisend. Die Anamnese sollte den Schmerzcharakter und die Entstehung explorieren und Miktionssymptome mit berücksichtigen. Eine Reihe klinischer Anhaltspunkte erleichtern die Diagnosestellung; diese sind in Tabelle 1 zusammengefasst. Die Injektion eines Lokalanästhetikums in den Samenstrang (zum Beispiel 5-10 ml Lido- cain) kann analgetisch sinnvoll sein und die Befunderhebung erleichtern.

Hydatidentorsion: „blue dot sign“ beachten. Die Klinik einer stilgedrehten Hydatide testis weist eine der Hodentorsion ähnliche Klinik auf und tritt zumeist bei Jungen im frühen Schulalter auf. Im Frühstadium vor Schwellung der Skrotalhüllen kann die hämorrhagisch infarzierte Hydatide als „blue dot sign“ durch die Skrotalhaut schimmern und als harte Resistenz palpabel sein.

Die primäre Orchitis betrifft ausschließlich den Hoden. Im klinischen Alltag tritt sie im Rahmen einer Mumps-Orchitis auf und ist gekennzeichnet durch einen (druck-)dolenten Hoden bei unauffälligem Nebenhoden.

Hodentrauma: Tunika des Hodens intakt? Die klinische Untersuchung beim stumpfen Hodentrauma gibt einen ersten Anhalt über Lokalisation und Ausmaß des Traumas, insbesondere, ob die Integrität des Nebenhodens und der Tunika des Hodens erhalten ist, was die weitere Therapiestrategie entscheidet [5].

\section{Apparative und Labordiagnostik}

Urinstatus, Sonografie (Abb. 1,2) und ggf. farbcodierte Duplexsonografie (FCDS) (Abb. 3) stellen wichtige Eckpfeiler für die Notfalldiagnostik dar. Tabelle $\mathbf{2}$ gibt die zu erwartenden Befunde wieder.

\section{Tabelle 1}

Anhaltspunkte zur klinischen Differenzialdiagnose zwischen Hodentorsion und akuter Epididymitis.

\begin{tabular}{|l|l|l}
\hline & Hodentorsion & Epididymitis \\
\hline Alter & vorwiegend bis zur Pubertät & vorwiegend ab Pubertät \\
\hline Symptome eines Harnwegsinfekts & nein & ja \\
\hline initialer Befund & elastisch fixierter Hodenhochstand, & Nebenhoden deutlich verdickt und hart, \\
& Nebenhoden an atypischer Stelle & Hoden unauffällig \\
\hline Beginn & perakut & über einige Stunden \\
\hline peritonealer Reiz (Erbrechen) & ja, gelegentlich & nein \\
\hline Hodenlage & Hochstand & normal \\
\hline Prehn'sches Zeichen & keine Abnahme des Schmerzes \\
\hline
\end{tabular}




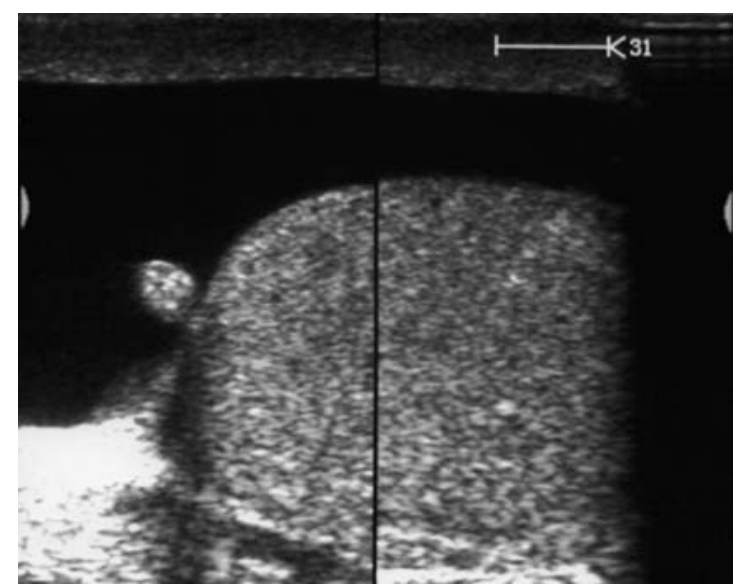

Abb. 1 Sonografisches Bild einer Hydatidentorsion mit Begleithydrozele.

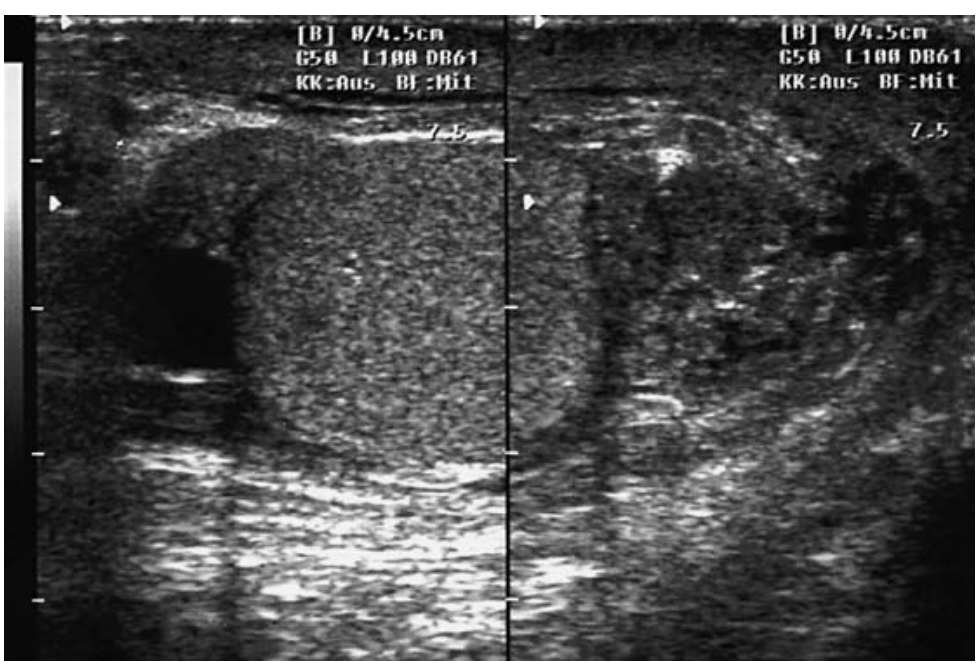

Abb. 2 Sonografisches Bild einer Nebenhodenschwanz-Epididymitis bei homogenem Hodenparenchym.

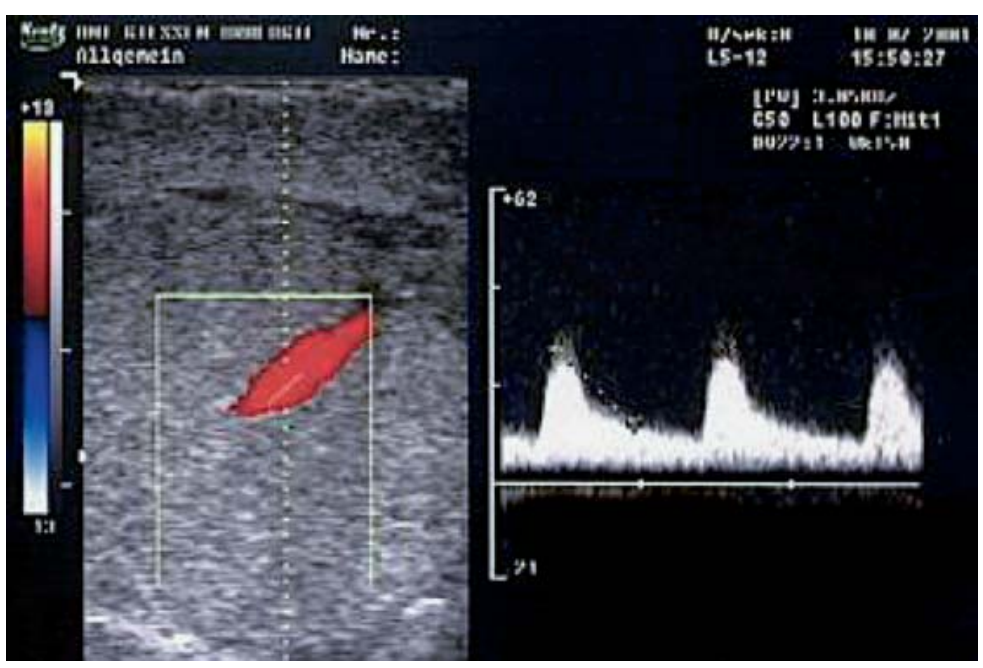

Abb. 3 Farbduplexsonografie bei intakter intratestikulärer arterieller Perfusion.
Von zentraler Bedeutung ist, dass trotz aller klinischen, labordiagnostischen und apparativen Hilfen eine eindeutige Diagnose nicht immer möglich ist. Dies lässt sich mit dem Einsatz der FCDS beispielhaft belegen. Einerseits werden in einzelnen Untersuchungen dieser Methode in der Hand des geübten Untersuchers eine Sensitivität und Spezifität von über 95\% zugeschrieben [6], andererseits zeigen diese Zahlen, dass im Einzelfall Fehldiagnosen zum Beispiel durch erhaltene arterielle Perfusion bei partieller Torsion nicht ausgeschlossen werden können. Da eine nicht rechtzeitig erfolgte Detorquierung einer Hodentorsion eine konsekutive Hodenatrophie zur Folge hat, muss der Stellenwert der FCDS relativiert werden. Bei unsicherer oder zweifelhafter Differenzialdiag-
nose Hodentorsion oder Entzündung ist die soforti-
ge operative Hodenfreilegung absolut indiziert.

Diagnose der Mumps-Orchitis: Serummarker. Bei klinischem Verdacht beweisen IgM-Antikörper gegen das Mumpsvirus die Diagnose.

\section{Therapie}

Akute Epididymitis: erregergerechte Antibiose. Die Therapie der akuten Epididymitis ist in erster Linie antimikrobiell und richtet sich nach der vermuteten Ätiologie (Tab. 3) [7]. Für die durch Neisseria gonorrhoeae verursachte Epididymitis existieren keine Empfehlungen. Hier ist zu beachten, dass auch in Deutschland eine hohe Resistenz dieses Keims gegenüber Fluorchinolonen (Ciprofloxacin, Ofloxacin) besteht. Ggf muss hier auf Ceftriaxon oder Cefixim zurückgegriffen werden [8]. Problematisch bleibt auch die optimierte Therapie bei unbekannter Erregerlage, wie sie in der klinischen Routine häufig vorkommt. Hier stehen Fluorchinolone der zweiten oder dritten Generation zur Verfügung, die eine gute Wirksamkeit gegenüber den meisten relevanten Erregern sowie eine hervorragende Gewebe- und Seminalplasmapenetration aufweisen.

Bei erhöhten Restharnmengen sollte ein suprapubischer Katheter platziert werden. Zahlreiche antiphlogistische (zum Beispiel nicht steroidale Antiphlogistika) und physikalische (zum Beispiel Hochlagerung und Kühlung) Maßnahmen sind weit verbreitet, jedoch in ihrer Wirksamkeit nicht prospektiv evaluiert. In 10-20\% tritt eine Abszedierung im Bereich des Nebenhodens auf, die eine operative Sanierung in Form einer Epididymektomie oder Semicastratio erforderlich macht. 


\section{Tabelle 2}

Erweiterte Diagnostik zur Differenzialdiagnose des akuten Skrotums.

\begin{tabular}{|c|c|c|c|c|}
\hline & Hodentorsion & Hydatidentorsion & Epididymitis & primäre Orchitis \\
\hline Urinbefund & unauffällig & unauffällig & $\begin{array}{l}\text { Erythrozyten, Leukozyten, } \\
\text { Bakterien }\end{array}$ & unauffällig \\
\hline $\begin{array}{l}\text { Sonografie } \\
\text { (häufige Befunde) }\end{array}$ & $\begin{array}{l}\text { variabel! } \\
\text { initial: Nebenhoden unauffällig, } \\
\text { Hodengewebe ggf. aufgelockert } \\
\text { nach } 4 \text { Stunden: testikuläre } \\
\text { Strukturveränderungen, } \\
\text { Bild wie bei Epididymitis }\end{array}$ & $\begin{array}{l}\text { Hoden und Nebenhoden } \\
\text { homogen, ggf. Darstellung } \\
\text { der Hydatide } \\
\text { Begleithydrozele }\end{array}$ & $\begin{array}{l}\text { Hoden homogen, } \\
\text { Nebenhoden verdickt, } \\
\text { aufgelockert } \\
\text { echoarme Areale: Abszess? }\end{array}$ & $\begin{array}{l}\text { Nebenhoden unauffällig } \\
\text { Hoden: ggf. aufge- } \\
\text { lockertes Parenchym }\end{array}$ \\
\hline $\begin{array}{l}\text { FCDS } \\
\text { (farbcodierte } \\
\text { Duplexsonografie) }\end{array}$ & $\begin{array}{l}\text { initial: keine intratestikuläre } \\
\text { arterielle Perfusion } \\
\text { Seitendifferenz } \\
\text { Cave: erhaltene Perfusion bei } \\
\text { subtotalen Torsionen } \\
\text { nach } 4-6 \text { h: FCDS nicht mehr } \\
\text { verwertbar }\end{array}$ & $\begin{array}{l}\text { intratestikuläre arterielle } \\
\text { Perfusion unauffällig }\end{array}$ & $\begin{array}{l}\text { intratestikuläre arterielle } \\
\text { Perfusion } \\
\text { Nebenhoden mit } \\
\text { verstärkter Perfusion }\end{array}$ & $\begin{array}{l}\text { intratestikuläre } \\
\text { arterielle (und venöse) } \\
\text { Perfusion verstärkt }\end{array}$ \\
\hline
\end{tabular}

\section{Tabelle 3}

Initiale antimikrobielle Therapie der Epididymitis gemäß EAU-Leitlinien.

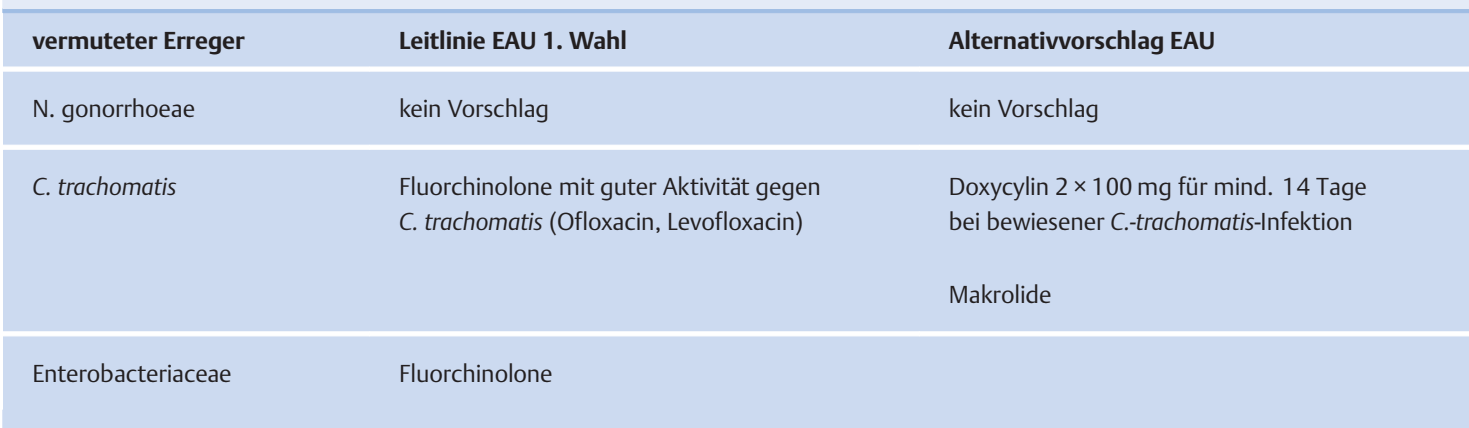

Hodentorsion: notfallmäßige operative Versorgung. Unbehandelt führt die Hodentorsion zu einer hämorrhagischen Infarzierung des Hodens mit sekundärem arteriellem Perfusionsstopp. Die möglichst frühzeitige operative Detorquierung mit Orchidopexie ist daher essenziell. Dies bedeutet in der präklinischen Situation für den Notarzt, dass bei entsprechendem Verdacht die umgehende Einweisung in eine urologische oder chirurgische Klinik absolut erforderlich ist. Bei partieller Torquierung kann auch nach der 6-Stunden-Grenze der Hoden durch operative Intervention noch gerettet werden. In der operativen Abteilung erfolgt anschlie- ßend oder in zweiter Sitzung die Orchidopexie der Gegenseite. Eine manuelle Detorquierung (Drehung des Hodens nach lateral) in der akuten Notfallsituation ist gelegentlich möglich, eine operative Freilegung ist aber trotzdem erforderlich.

\section{Hydatidentorsion: konservative Therapie möglich.}

Sollte eine Hodentorsion mit Sicherheit ausgeschlossen sein, kann eine Hydatidentorsion auch symptomatisch mit Analgetika oder Antiphlogistika therapiert werden. Die Indikation zur Freilegung im Intervall stellt sich bei zunehmender lokaler Symptomatik (Schwellung, Be- 
schwerden). Auch hier gilt der Leitsatz, im Zweifel immer freizulegen. Dies erfordert in der präklinischen Situation durch den Notarzt das gleiche Vorgehen wie bei der Hodentorsion.

Eine standardisierte Therapie der primären MumpsOrchitis existiert nicht. Einzelne Arbeitsgruppen berichten über den Einsatz von GnRH-Agonisten zur Protektion der Spermatogenese oder über den systemischen Einsatz von Alpha-2b-Interferon, um eine Hodenatrophie zu verhindern, was aber nicht zuverlässig gelingt [2].

Stumpfes Hodentrauma: definierte Indikationen zur Freilegung. Während das penetrierende Hodentrauma in jedem Fall eine operative Revision erfordert, existieren beim stumpfen Hodentrauma definierte Indikationen zur Freilegung [5]:

- Verdacht auf Hodenruptur

- größere Hämatozele

- unklarer Befund

Die operative Versorgung der Hodenruptur umfasst die Exzision nekrotischen Hodengewebes und Verschluss der rupturierten Tunica albuginea testis. In der Regel ist die Prognose hinsichtlich Hodenerhalt und endokriner Hodenfunktion gut.

Als Anhaltspunkt für die Hämatozele gilt, dass ein konservativer Therapieversuch statthaft ist, wenn sie kleiner als 3-mal so groß wie der kontralaterale Hoden imponiert. Bei größeren Hämatozelen sollte die primäre operative Versorgung angestrebt werden, da ein verzögertes Vorgehen oft eine Versorgung im Intervall erfordert und diese eine höhere Rate an Orchiektomien bedingt. Bei früher Intervention ist ein Hodenerhalt in über 90\% der Fälle möglich, bei verzögerter Operation nur noch in ca. $50 \%$ [5].

\section{Flankenschmerz}

\section{Symptomatologie und Differenzialdiagnosen}

Stellt sich ein Patient mit Flankenschmerzen notfallmäßig vor, so ist im Regelfall von starken oder stärksten Schmerzen und zumeist auch von einem akuten Schmerzbeginn oder einer raschen Verschlechterung einer zuvor stabilen Situation auszugehen. Weiterhin bestehen häufig Begleitsymptome, wie zum Beispiel Übelkeit/Erbrechen oder Fieber.
Folgende Fragestellungen sollten in der Schmerzanamnese eines Patienten mit Flankenschmerzen immer geklärt werden, da sie für die richtige Differenzialdiagnose und damit auch die richtigen Therapieschritte entscheidend sein können [9]:

- Schmerzlokalisation und -ausstrahlung: Höhe und Ausstrahlung des Flankenschmerzes können verhältnismäßig genau Anhalt für die Lokalisation des Schmerzauslösers (zum Beispiel eines Harnsteins) im Verlauf des oberen Harntrakts geben (Abb. 4). So können die Kolikschmerzen beim distalen Harnleiterstein bis in das äußere Genitale ausstrahlen.

- Schmerzcharakter: Während der viszerale Parenchymschmerz eher auf eine Druckerhöhung im Hohlsystem bei Obstruktion oder eine entzündliche Affektion des Nierenparenchyms hinweist, deuten auf die Bauch- oder Thoraxwand projizierte parietale Schmerzen möglicherweise eher auf ein retroperitoneales Geschehen hin, zum Beispiel bei Blutung oder retroperitonealem Abszess. Es ist sicherlich zu beachten, dass es dem Patienten in der akuten Schmerzsituation schwer fällt, den Schmerzcharakter präzise zu beschreiben.

- Schmerzverlauf: Das Schmerzkontinuum ist vom klassischen, kolikartigen Verlauf zu unterscheiden. Die glattmuskulären Kontraktionen, die letztendlich auslösend für den Kolikschmerz sind, können wenige Minuten bis zu Stunden im Status colicus dauern.

- Schmerzintensität: Die Dokumentation ist insbesondere für die Beurteilung des Therapieerfolgs wichtig, es empfiehlt sich die Verwendung möglichst objektiver Messinstrumente, zum Beispiel einer visuellen Analogskala.

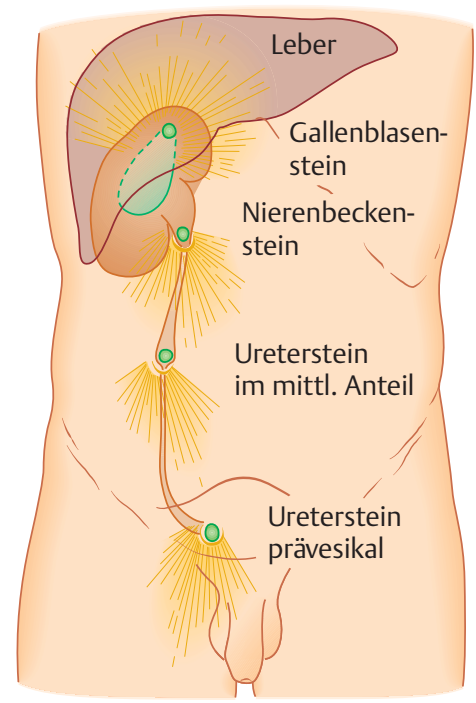

Abb. 4 Schmerzausstrahlung bei verschiedenen Steinlokalisationen (Quelle: Sökeland, Haßdenteufel, Krieger, Lutz. Urologie für Pflegeberufe. Thieme 2000). 
- Begleitsymptome sind in jedem Fall zu erfragen und zu dokumentieren. Während Übelkeit, Meteorismus, Subileus und Erbrechen Folge jeder peritonealen Reizung sein können, geben Entzündungszeichen bis hin zu septischen Zustandsbildern zum Beispiel Hinweis auf eine infizierte Harnstauungsniere und erfordern sofortiges Handeln.

- Komorbiditäten können Hinweise auf Ursachen insbesondere akuter Schmerzereignisse geben (Diabetes, Vorhofflimmern, Thromboembolie-Anamnese, zystische Nierenerkrankungen).

Die wichtigsten Differenzialdiagnosen des Flankenschmerzes sind - eingeteilt nach urologischen und nicht urologischen Ursachen - in Tabelle $\mathbf{4}$ wiedergegeben.

\section{Obstruktion des oberen Harntrakts}

Jede Obstruktion des normalen Urinabflusses aus der Niere hat biochemische, immunologische, hämodynamische und funktionelle Veränderungen zur Folge. Es kommt eine Kaskade in Gang, in der Angiotensin II, Zytokine und Wachstumsfaktoren zu Entzündung, tubulo-interstitieller Fibrose und letztlich Tubuluszellapoptose führen. Die rasche Beseitigung einer Obstruktion sollte immer das primäre Therapieziel bei Harntransportstörungen sein. Die Erholung der Niere nach Behebung einer Obstruktion hängt von der Dauer und dem Ausmaß der Harntransportstörung, dem Alter des Patienten und der Ausgangsnierenfunktion ab.

Ätiologie und Ursachen. Ursächlich für den akuten Flankenschmerz ist zumeist eine plötzliche Verlegung des Ureterlumens durch

- einen Stein

- Blutkoagel bei Blutung im Bereich des oberen Harntrakts

- abgehende Gewebeanteile (zum Beispiel Tumorgewebe bei Neoplasmen im Bereich von Niere oder Nierenbecken oder auch Nierengewebe bei Papillennekrosen, die bei Diabetikern oder analgetikainduzierter Nephropathie vorkommen)

Chronische Obstruktionen des oberen Harntrakts, zum Beispiel durch Harnleitertumoren, Tumorkompression von außen oder narbige Ureterstrikturen, bleiben meist symptomlos.

Diagnostik. Bei jedem Patienten, der sich mit akuten Flankenschmerzen vorstellt, ist eine Harntransportstörung mittels Ultraschall auszuschließen bzw. nach-

\section{Tabelle 4}

Differenzialdiagnosen bei Flankenschmerz.

\begin{tabular}{|c|c|}
\hline Urogenitaltrakt & $\begin{array}{l}\text { - Harnleiterkolik bei Urolithiasis } \\
\text { - Obstruktion des oberen Harntrakts (Schmerzen meist nur } \\
\text { bei akuter Obstruktion), unter Umständen mit Fornixruptur } \\
\text { - Pyelonephritis } \\
\text { - Nierenabszess } \\
\text { - Niereninfarkt oder Nierenvenenthrombose } \\
\text { - } \text { akute Einblutung einer Nierenzyste oder eines Tumors oder } \\
\text { auch nach extrakorporaler Stoßwellenbehandlung } \\
\text { - retroperitoneale Prozesse (Blutung oder Abszess) } \\
\text { - Tumoren der Niere oder des Retroperitoneums } \\
\text { (seltene Schmerzursache) }\end{array}$ \\
\hline Andere Ursachen & $\begin{array}{l}\text { - vertebrogen-radikuläre Schmerzen } \\
\text { - Rippenfrakturen, vor allem dorsal } \\
\text { - Ausstrahlung bei Pankreatitis (beidseits) oder Cholezystitis } \\
\text { (rechts) } \\
\text { - Aortendissektion/Aneurysmaruptur (auch gedeckt) }\end{array}$ \\
\hline
\end{tabular}

zuweisen. Die Diagnose wird bei entsprechenden apparativen Voraussetzungen in der Praxis oder Klinik gestellt und sollte als Minimalanforderung folgende diagnostischen Maßnahmen nach sich ziehen, um eine Therapieentscheidung treffen zu können [10]:

- vollständige körperliche Untersuchung und Anamnese

- Urinstatus (Mittelstrahlurin beim Mann, Katheterurin bei der Frau, ggf. Blasenpunktionsurin beim Kleinkind) zum Nachweis oder Ausschluss eines Harnwegsinfekts oder einer Hämaturie

- Temperaturmessung

- Labor mit kleinem Blutbild, CRP, Elektrolyten und Nierenretentionswerten, Gerinnung

Spezielle Therapiemaßnahmen. Unabhängig von der Ursache des Harnaufstaus besteht bei bestimmten Konstellationen eine absolute Indikation zur Entlastung einer Harnstauungsniere (siehe Infobox).

Indikationen zur Entlastung einer Harnstauungsniere

- Fieber oder Sepsiszeichen (infizierte Harnstauungsniere)

- laborchemische Zeichen einer progredienten Einschränkung der Gesamtnierenfunktion

- nicht beherrschbare Schmerzen/Status colicus

- Generell gilt: Im Zweifel oder bei grenzwertigen Befunden ist eine gestaute Niere zu entlasten. 


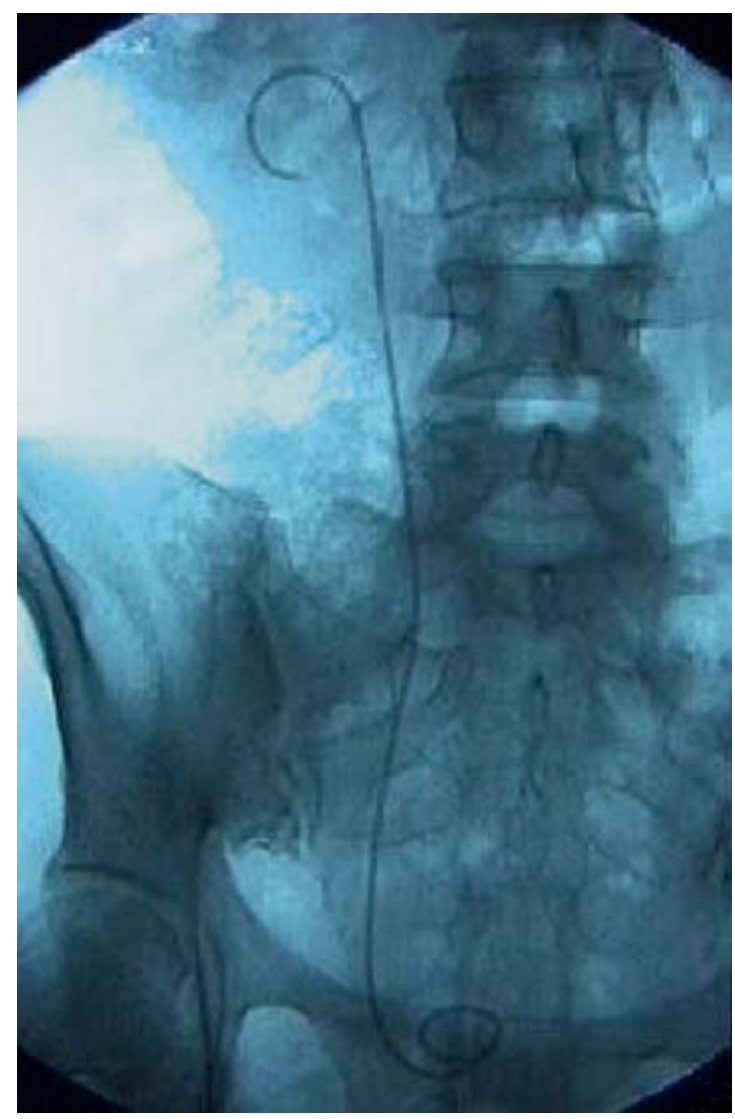

Abb. 5 Abdomen-Leeraufnahme bei einliegender Doppel---Schiene.

Die Entlastung einer Harnstauungsniere kann auf 2 Wegen erfolgen: entweder retrograd-transurethral oder perkutan-transrenal.

- Bei retrograd-transurethralem Vorgehen wird über eine Zystoskopie das Harnleiterostium sondiert, das Abflusshindernis mit einem Draht oder einem Ureterenkatheter passiert und der Harnleiter mittels eines Doppel-J- oder Mono-J-Katheters geschient und somit ein Urinabfluss ermöglicht. Der Doppel-Joder Doppel-Pigtail-Katheter bietet den Vorteil, dass er in der Blase versenkt ist, er kann jedoch leichter obliterieren. Der Mono-J lässt eine seitengetrennte Beurteilung der Nierenfunktion zu und kann manuell frei gespült werden, ist jedoch durch die Urethra nach außen ausgeleitet und erfordert zur Fixation die gleichzeitige Einlage eines Ballon-Dauerkatheters (Abb. 5).

- Die perkutan transrenale Anlage einer Nephrostomie erfolgt sonografisch und/oder radiologisch kontrolliert in Lokalanästhesie in Bauchlage. Diese Intervention ist dann notwendig, wenn eine retrograde Entlastung nicht möglich ist (zum Beispiel bei kompletter Obstruktion durch großen Stein), ist aber

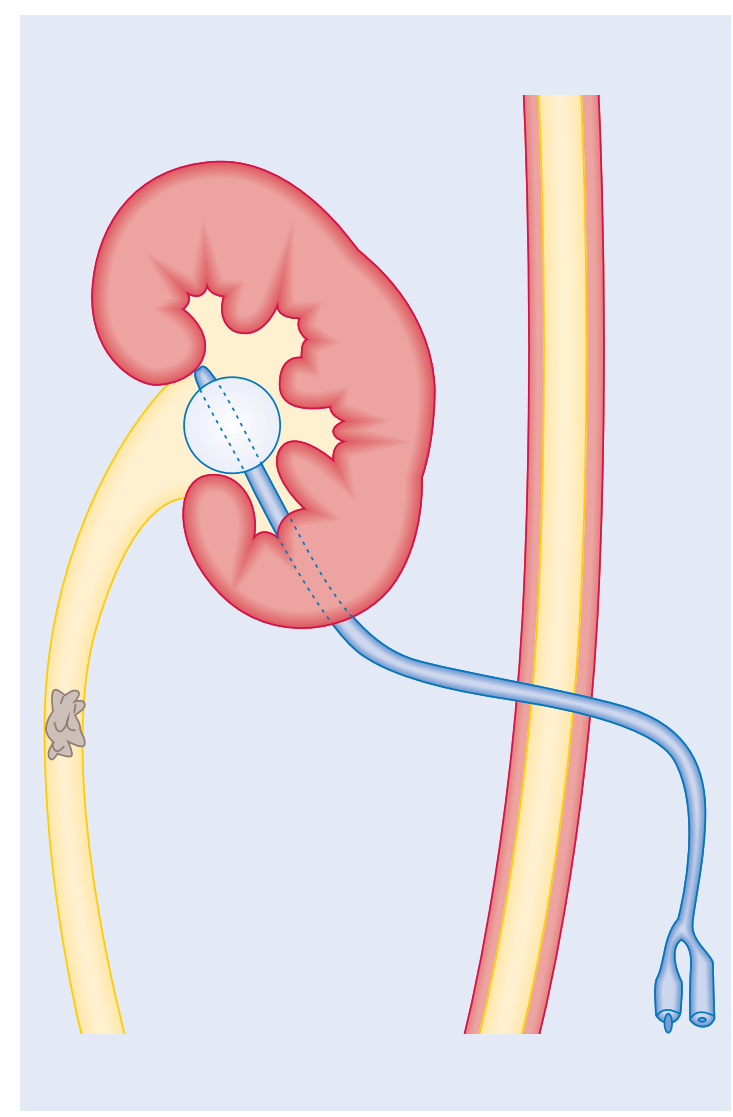

Abb. 6 Schemazeichnung einer einliegenden, perkutanen Nephrostomie bei Harnleiterobstruktion durch Stein.

auch eine Option der ersten Wahl, insbesondere dann, wenn der Patient einer transurethralen Manipulation zurückhaltend gegenübersteht (Abb.6).

Sowohl bei der retrograden als auch bei der perkutanen Entlastung kann die Intervention mit einer Kontrastmitteldarstellung des Nierenhohlraumsystems verbunden werden. So kann die Lokalisation und mit Einschränkungen auch die Ursache (schattengebender Stein vs. nicht schattengebender Stein oder Verdacht auf Tumor oder Striktur) der Obstruktion festgestellt werden.

Spezielle Diagnostik und Therapie bei Harnleiterkolik werden weiter unten abgehandelt.

\section{Infektionen}

Ätiologie und Ursachen. Auch Infektionen im Bereich der oberen ableitenden Harnwege und der Nieren können für Flankenschmerzen verantwortlich sein, die den Patienten dazu veranlassen, einen ärztlichen Notdienst 


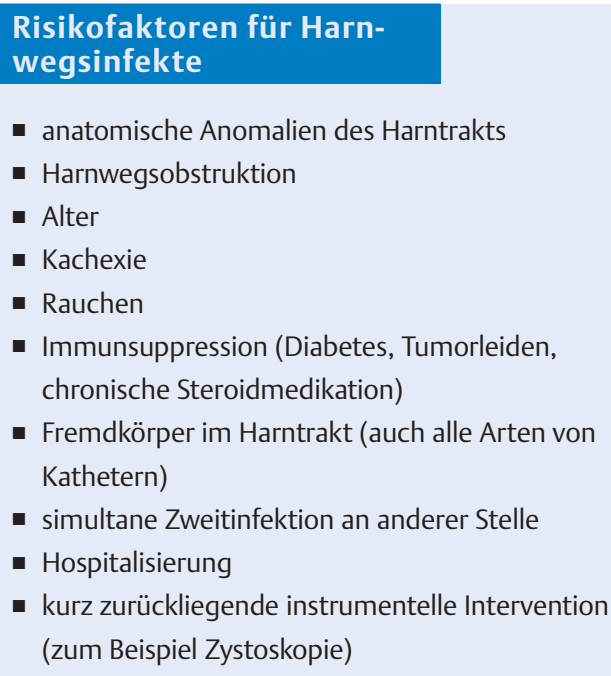

- anatomische Anomalien des Harntrakts

- Harnwegsobstruktion

- Alter

- Kachexie

- Rauchen

- Immunsuppression (Diabetes, Tumorleiden, chronische Steroidmedikation)

- Fremdkörper im Harntrakt (auch alle Arten von Kathetern)

- simultane Zweitinfektion an anderer Stelle

- Hospitalisierung

- kurz zurückliegende instrumentelle Intervention (zum Beispiel Zystoskopie)

zu konsultieren. Die Pyelonephritis ist in den meisten Fällen eine aszendierende Infektion, häufig mit Darmkeimen. In 30\% der Harnwegsinfekte bei Frauen und in $5 \%$ bei Männern liegen Risikofaktoren vor, die für Harnwegsinfekte und somit auch für Pyelonephritiden prädisponieren [11] (siehe Infobox).

Diagnostik. Das klinische Bild der Nierenbeckenentzündung kann von leichten Flankenschmerzen bis zu schwer septischen, akut lebensbedrohlichen Verläufen reichen.

Die präklinische Diagnostik sollte in jedem Fall eine symptomorientierte Anamnese, eine gründliche körperliche Untersuchung mit Bestimmung der Körpertemperatur sowie, falls verfügbar, eine Urinstix-Untersuchung beinhalten. Richtungsweisend für eine Pyelonephritis in der Anamnese ist neben den oben angegebenen Risikofaktoren insbesondere eine simultan bestehende Zystitis mit den hierfür typischen Miktionsbeschwerden.

In der körperlichen Untersuchung fällt insbesondere der dumpfe, manchmal pochende Flankenschmerz auf, der durch Druck oder Klopfen auslösbar oder verstärkbar ist. Lokale Wärme wird meist als angenehm empfunden. Eine Leukozyturie oder ein Nitrit-positiver Urin machen eine Harnwegsinfektion sehr wahrscheinlich.

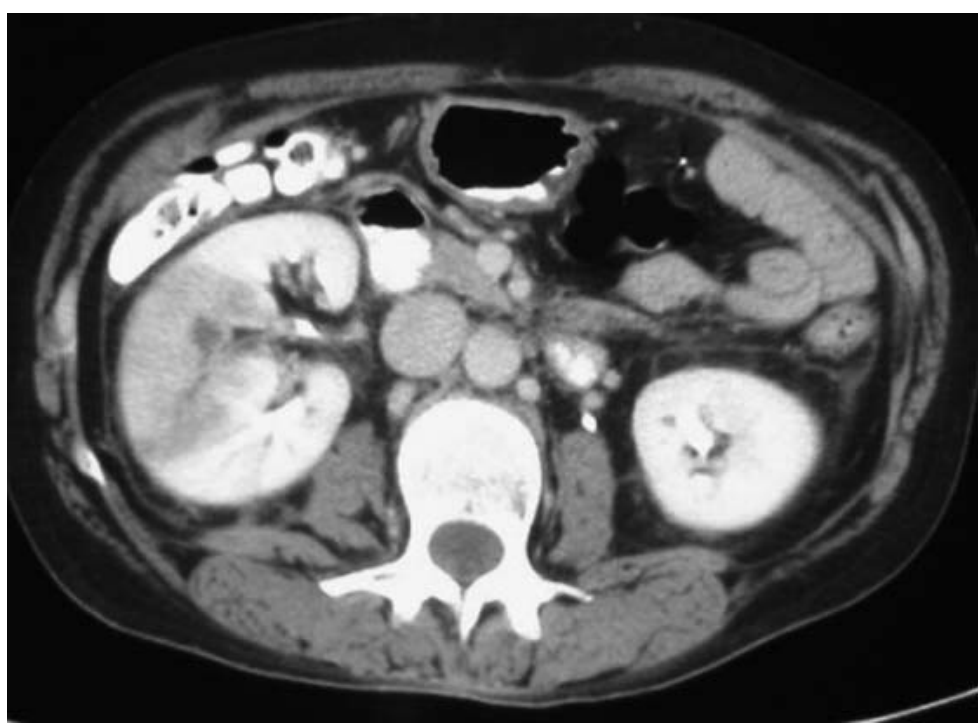

Abb. 7 Typische KM-verstärkte Computertomografie bei Pyelonephritis rechts mit beginnender Einschmelzung.

Spezielle Therapiemaßnahmen. Kausale Therapie der Pyelonephritis ist die kalkulierte antibiotische Therapie für 7-10 Tage (nach Uringewinnung für die Kultur), typischerweise mit Cotrimoxazol, einem Gyrasehemmer oder einem Cephalosporin (unter Beachtung der jeweiligen Kontraindikationen). Diese ist ggf. bei schweren Verläufen stationär und i.v. durchzuführen. Außerdem ist auf ausreichende Flüssigkeitssubstitution und eine adäquate Schmerztherapie zu achten.

Bei mangelnder Entfieberung ist die Resistenzlage zu prüfen, ggf. das Antibiotikum umzusetzen und bei Verdacht auf Reflux temporär ein Dauerkatheter anzulegen. Des Weiteren ist bei komplizierten Verläufen die bildgebende Diagnostik zu wiederholen oder ggf. mittels Schichtbildgebung zu intensivieren.

Nierenabszesse (Abb. 7) sind, wenn keine Harnstauungsniere vorliegt, im Normalfall unter antibiotischer Therapie zu beherrschen, bei fehlendem Ansprechen auf die Therapie kann eine Abszessdrainage (Sonooder CT-gesteuert) notwendig sein, sehr selten die sofortige Nephrektomie (Abb. 8) [11].

Bei Verdacht auf obstruktive Pyelonephritis ist die betroffene Niere zu entlasten!

Die weiterführende klinische Diagnostik muss dann zumindest ein Labor, eine Ultraschalluntersuchung sowie eine Urinkultur umfassen. Je nach Beschwerdebild, auf jeden Fall bei hohem Fieber oder Zeichen der Sepsis ist die stationäre Aufnahme indiziert. 


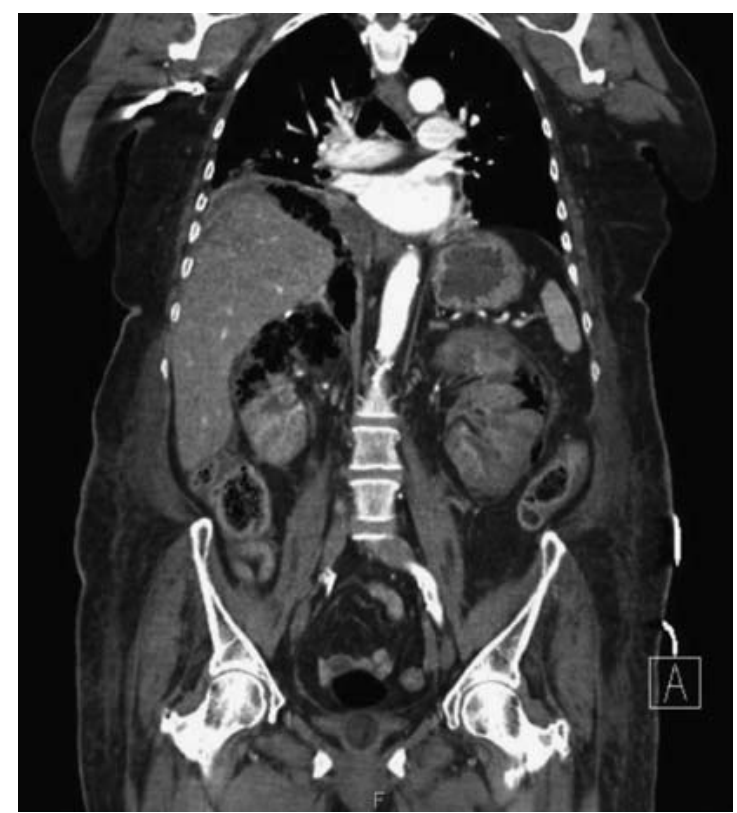

Abb. 8 Schwere beidseits gangränös abszedierende Nephritis, Indikation zur Nephrektomie bei schwerster Sepsis mit Organversagen.

\section{Urolithiasis/Kolik}

Ätiologie. Harnsteine, die sich im Nierenbeckenkelchsystem befinden, können bis zu einer beträchtlichen Größe symptomarm oder sogar asymptomatisch bleiben. Tritt jedoch ein Stein in den Ureter ein, so kommt es in den meisten Fällen zu einem Harnaufstau (Abb. 9) und zu Harnleiterkoliken.

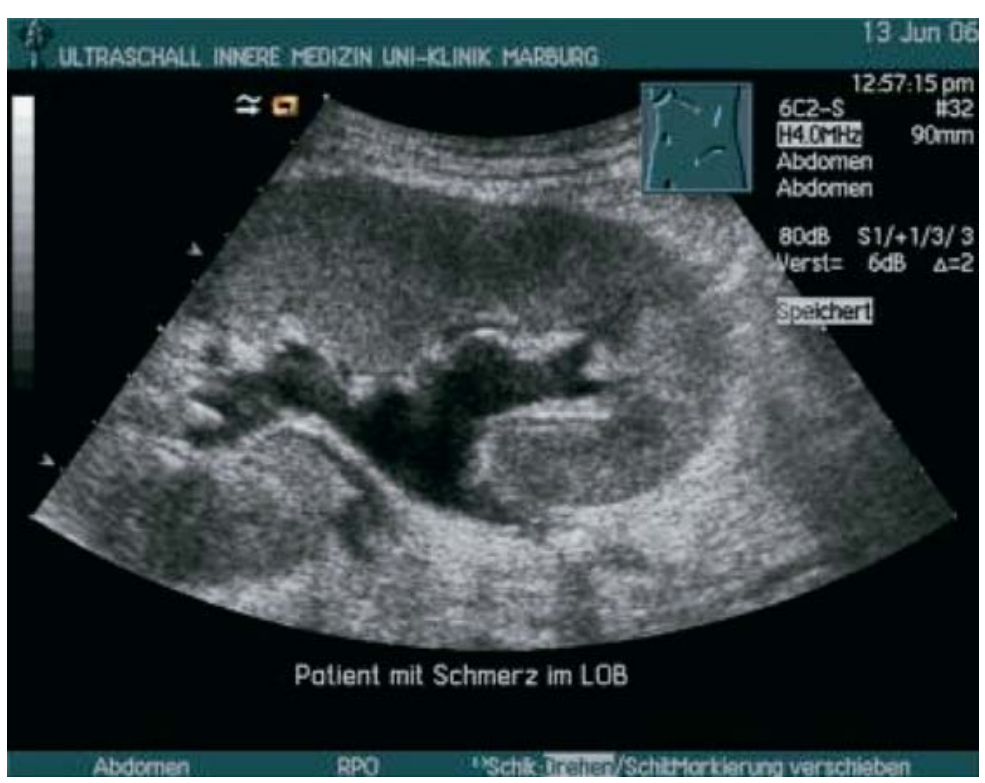

Abb.9 Sonografisches Bild eines Harnaufstaus mit proximalem Harnleiterstein.
Klinik. Die typische Kolik beginnt plötzlich in Form krampfartiger, anfallsweise auftretender, wehenartiger Schmerzen im Nierenlager und entlang des Harnleiterverlaufs. Sie kann Minuten bis Stunden dauern [12]. Eine Ausstrahlung in die Blasenregion, in den Genitalbereich oder die Oberschenkelinnenseite in Abhängigkeit von der Steinlokalisation ist typisch (Abb. 4). Für den Kolikschmerz sind vor allem 2 Faktoren verantwortlich:

- Dilatation des obstruierten Hohlsystems mit Druckerhöhung und Dehnung der Schmerzrezeptoren in Nierenbecken und -kelchen

- lokale Irritation der Ureterwandung mit Ödembildung und Ausschüttung von Schmerzmediatoren

Diagnostik. Anamnestisch sind irritative Miktionsbeschwerden oder auch eine Makrohämaturie typisch. Fieber oder Schüttelfrost deuten auf eine begleitende Infektion oder eine infizierte Harnstauungsniere hin und sind als Alarmsignal zu werten. Typischerweise verschafft leichte körperliche Bewegung dem Patienten/der Patientin eher Erleichterung. Dies ist bei einer vertebromuskulären Ursache oder beim akuten Abdomen mit Peritonismus nicht der Fall und ermöglicht eine gewisse differenzialdiagnostische Eingrenzung.

Die körperliche Untersuchung erbringt neben dem typischen Klopfschmerz im Flankenbereich häufig einen ausgeprägten Meteorismus, der durch die begleitende peritoneale Reizung bedingt ist. Der Ureter liegt ja dem dorsalen Peritoneum parietale direkt an [12]. In der präklinischen Diagnostik kann des Weiteren eine Mikrohämaturie im Urinstix hinweisgebend sein (Tab. 5).

\begin{tabular}{|c|c|}
\hline \multicolumn{2}{|l|}{ Tabelle 5} \\
\hline \multicolumn{2}{|c|}{ Notfalldiagnostik beim Kolikpatienten. } \\
\hline Labor (Blut) & $\begin{array}{l}\text { Kreatinin/Harnstoff, Harnsäure, } \\
\text { Elektrolyte inkl. ionisiertem Kalzium, } \\
\text { Blutbild, CRP, Gerinnung }\end{array}$ \\
\hline Labor (Urin) & Urinstatus, Urinkultur \\
\hline Bildgebung & $\begin{array}{l}\text { Sonografie, Spiral-CT des Abdomens } \\
\text { nativ, alternativ Ausscheidungs- } \\
\text { urogramm }\end{array}$ \\
\hline $\begin{array}{l}\text { weitere Diagnostik } \\
\text { nach Akuttherapie }\end{array}$ & $\begin{array}{l}\text { Harnsteinanalyse, Sammelurinanalyse } \\
\text { auf steinbildende Substanzen, metabo- } \\
\text { lische Diagnostik auf prädisponierende } \\
\text { Erkrankungen }\end{array}$ \\
\hline
\end{tabular}


Spezielle Therapiemaßnahmen. Im Vordergrund steht bei der Akuttherapie der Nierenkolik, die sich für den Patienten als Vernichtungsschmerz manifestieren kann, eine adäquate analgetische Therapie. Prinzipiell gelten - unter Beachtung der jeweiligen Kontraindikationen und nach Allergieanamnese - die Grundsätze des WHO-Stufenschemas zur Schmerztherapie (Abb. 10).

Häufig ist mit Nichtopioid-Analgetika bereits rasche Schmerzfreiheit zu erzielen. Im Vordergrund stehen hier Metamizol und nicht steroidale Antiphlogistika, die in den Empfehlungen der Fachgesellschaften als Therapeutika der ersten Wahl genannt werden. Zentral wirksame Analgetika sind seltener notwendig, sollten bei refraktären Schmerzen jedoch zum Einsatz kommen. Beim therapierefraktären Status colicus ist eine interventionelle Entlastung der betroffenen Niere (siehe oben) indiziert.

Die eigentliche kausale Therapie der Steinkolik, nämlich die Beseitigung des Harnsteins, fällt nicht mehr in den Bereich der Akuttherapie. Die verschiedenen Therapiemodalitäten von konservativ bis interventionell sind in Tabelle 6 kurz zusammengefasst.

Die offene Steinchirurgie stellt aufgrund der mittlerweile ausgezeichneten Visualisierung und Desintegrationsmöglichkeiten über immer kleinere, atraumatische Endoskope eine Ausnahme dar. Aufgrund höherer Steinfreiheitsraten in einer Therapiesitzung wird die Endoskopie auch gegenüber der ESWL mehr und mehr favorisiert [12].

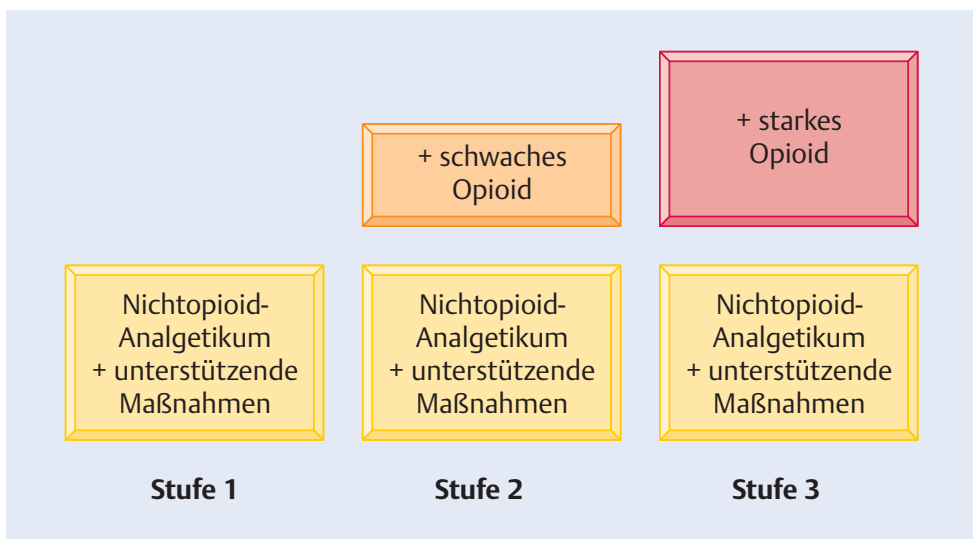

Abb. 10 WHO-Stufenschema der Schmerztherapie.

Seltene Differenzialdiagnosen. Weitere, seltene Differenzialdiagnosen des akuten Flankenschmerzes sind

- Niereninfarkt/hämorrhagische Infarzierung

- Einblutung in eine Nierenzyste/Zystenruptur, vor allem bei hereditäten zystischen Nierenerkrankungen

- spontane Nierenparenchymblutung oder Einblutung in einen Nierentumor, vor allem unter Antikoagulation

Diese Diagnosen können sicher nur durch entsprechende Bildgebung gestellt werden.

\section{Akuter Harnverhalt}

Ein akuter Harnverhalt ist gekennzeichnet durch das plötzliche schmerzhafte Unvermögen, die Harnblase zu entleeren.

\section{Tabelle 6}

Therapieoptionen bei Harnleiterstein

\begin{tabular}{|llc|}
\hline Verfahren & Indikation & Erfolg (\%) \\
\hline $\begin{array}{l}\text { konservative Maßnahmen, Spasmoanalgesie, } \\
\text { unterstützende medikamentöse Maßnahmen zur } \\
\text { Steinexpulsion (zum Beispiel Alphablocker, Steroide) }\end{array}$ & $\begin{array}{l}\text { Harnleitersteine }<8 \mathrm{~mm} \text {, vor allem mittleres } \\
\text { und unteres Drittel }\end{array}$ & $60-80$ \\
\hline $\begin{array}{l}\text { extrakorporale Stoßwellenlithotripsie (ESWL) in situ } \\
\text { (ohne auxiliäre Maßnahmen) }\end{array}$ & $\begin{array}{l}\text { vor allem proximale, partiell obstruierende Steine }<1 \mathrm{~cm}, \\
\text { keine wesentliche Harnstauung, asymptomatischer Patient }\end{array}$ & $80-90$ \\
\hline $\begin{array}{l}\text { ESWL mit auxiliären Maßnahmen } \\
\text { (Harnleiterschienung, perkutane Nephrostomie) }\end{array}$ & $\begin{array}{l}\text { obstruierende Steine, vor allem proximal, } \\
\text { Harntransportstörung, Koliken }\end{array}$ & $70-80$ \\
\hline $\begin{array}{l}\text { Ureterorenoskopie mit Steindesintegration } \\
\text { (Laser, pneumatisch) und/oder Extraktion }\end{array}$ & Steine jeder Größe und Höhe & $80-100$ \\
\hline
\end{tabular}




\section{Ursachen infravesikaler Obstruktion}

- mechanisch (führt zu Überdehnung und Dekompensation des Harnblasenmuskels)

- dynamisch-funktionell (neurologisch, psychisch, medikamentös: keine oder eingeschränkte Harnblasenmuskelkontraktion)

- seltener: vesikal bedingte Obstruktionen mit einer Verlegung des Blasenhalses, zum Beispiel durch Blasentumoren oder Harnblasensteine (Abb. 11 und 12)
Der akute Harnverhalt stellt einen sehr häufigen urologischen Notfall dar, welcher hauptsächlich bei Männern auftritt, bei Frauen ist dieser sehr selten. Die Inzidenz des akuten Harnverhalts beträgt bis zu 7/1000 Männern und zeigt aufgrund der sich ändernden Altersstruktur eine steigende Tendenz [13-15].

Die genauen Ursachen einer akuten Harnverhaltung sind unklar, Risikofaktoren wie zum Beispiel Alter > 70 Jahre, bestehende Miktionsprobleme etc. konnten identifiziert werden [16]. Mehrere unterschiedliche Mechanismen können einen akuten Harnverhalt zu bedingen (Tab. 7) [13-17].

Durch die zunehmende Füllung mit der plötzlichen Unfähigkeit, die Harnblase zu entleeren, entsteht ein zunehmender Blasendruck. Der Patient berichtet über einen dauernden, quälenden Harndrang, verbunden mit einem progredienten suprapubischen Schmerz und eventuell den Symptomen einer Überlaufinkontinenz mit tröpfchenweisem Urinverlust. Oft berichten die Patienten über eine länger vorliegende Harnblasenentleerungsstörung, zum Beispiel aufgrund einer bekannten benignen Prostatahyperplasie (Abb. 3). Klinisch wirkt der Patient unruhig, ist blass und schweißig.

Anamnestisch sollten Medikamente, die zu einer Blasenfunktionsstörung führen können (vor allem Anticholinergika und Psychopharmaka), genauso wie auslösende Trigger-Faktoren (Kälte, alkoholische Getränke) erfragt werden [17].

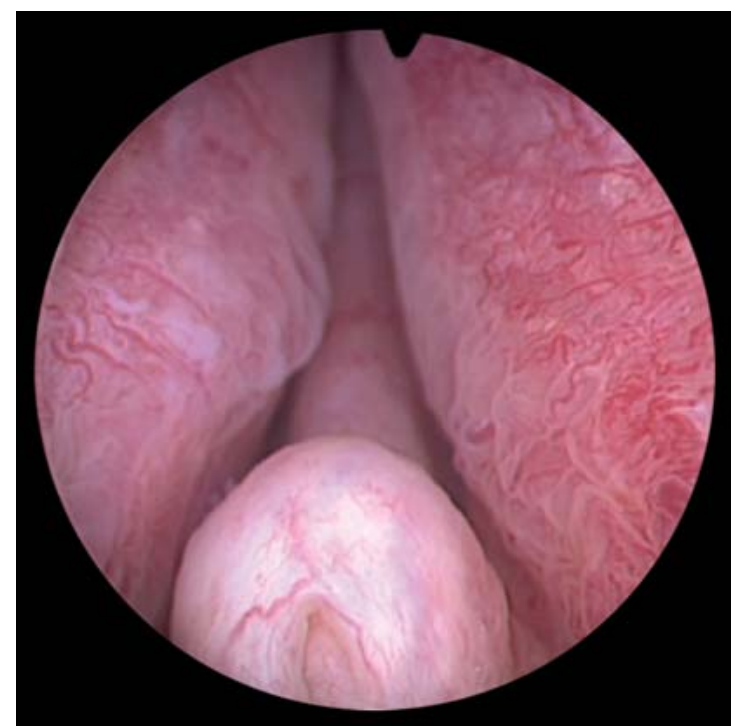

Abb. 13 Endoskopisches Bild einer deutlich vergrößerten Prostata mit prominenten, das Lumen obstruierenden Seitenlappen; in der Mitte ist der Samenhügel erkennbar (Quelle: Axel Hegele). 


\section{Tabelle 7}

Häufige Ursachen eines akuten Harnverhalts.

\begin{tabular}{lll}
\hline mechanische infravesikale Ursachen & funktionelle inrafvesikale Ursachen & vesikal bedingte Ursachen \\
\hline Prostatavergößerung & neurologisch: & Blasentumor \\
Prostatakarzinom & - Diskusprolaps (L1-L5) & Blasenstein(e) \\
akute Prostatitis & psychogen & Blasentamponade \\
Urethrastriktur & medikamentös: & Fremdkörper \\
Blasenhalsenge & - Psychopharmaka & \\
Meatusstenose & - vegetativ wirksame Medikamente & \\
Manipulationsfolge & & \\
(zum Beispiel iatrogen/autoerotisch ...) & & \\
\end{tabular}

Bei neurologischen Ursachen einer Harnverhaltung kann die beschriebene Symptomatik durch Störungen bzw. Ausfall der Blasensensibilität fehlen. Eine Besonderheit ist der spinale Schock, etwa nach einem schweren spinalen Trauma.

Bei der körperlichen Untersuchung kann der Unterbauch durch die massiv gefüllte Harnblase kugelförmig vorgewölbt sein. Bei der Palpation besteht ein deutlicher Druckschmerz dieser Region und die gefüllte Harnblase kann als prall elastische halbrunde Raumforderung imponieren.

Zusammen mit der Anamnese und dem klinischen Erscheinungsbild ist die Diagnose des akuten Harnverhalts meist sehr schnell eindeutig zu stellen.

Sollten Zweifel bestehen, kann die Sonografie Klarheit schaffen.

Therapie des akuten Harnverhalts: sofortige Entlastung der Harnblase - unabhängig von der vermuteten Ursache!

Die notfallmäßige Entlastung der Harnblase kann entweder mittels transurethraler Katheterisierung oder durch eine suprapubische Blasenpunktion erfolgen.

Hierbei gilt, dass im Notfall vor Ort die Durchführung einer transurethralen Katheterisierung deutlich praktikabler ist. Man sollte darauf achten, den Katheter unter sterilen Bedingungen (kommerzielle Einmalsets), in entspannter Rückenlage atraumatisch anzulegen, um eine Verletzung der Harnröhre/Prostata zu vermeiden.
Der sterile Einmalkatheterismus beseitigt allerdings meist die akuten Symptome nur kurzfristig, da es häufig bei erneuter Blasenfüllung wiederum zu einem Harnverhalt kommt, sodass die Einlage eines transurethralen Dauerkatheters und zeitnahe Abklärung der Ursache primär sinnvoller erscheint.

Gelingt die transurethrale Anlage eines Katheters nicht (zum Beispiel bei einer ausgeprägten Harnröhrenenge oder einem mechanischen Hindernis) sollte die suprapubische Anlage eines Katheters möglichst in einer urologischen Klinik/Einheit mit Möglichkeit der sonografischen Kontrolle erfolgen: Nach Setzen einer Lokalanästhesie wird die volle Harnblase mit einer Trokarnadel etwa 2 Querfinger oberhalb der Symphyse punktiert und hierüber ein Katheter in die Harnblase eingelegt (Abb. 14).

Nach Entlastung der Harnblase mittels Katheterisierung sind die akuten, unkomfortablen Symptome erfreulicherweise sehr rasch beseitigt und es kann eine weiterführende Diagnostik und später eine entsprechende definitive Therapie erfolgen [17-19].

\section{Makrohämaturie}

Als Makrohämaturie wird die vermehrte Ausscheidung von Erythrozyten über den Urin beschrieben, welches eine Rotfärbung des Urins zur Folge hat und mit dem Auge erkennbar ist. Die Makrohämaturie ist ein Symptom und kein Krankheitsbild. 


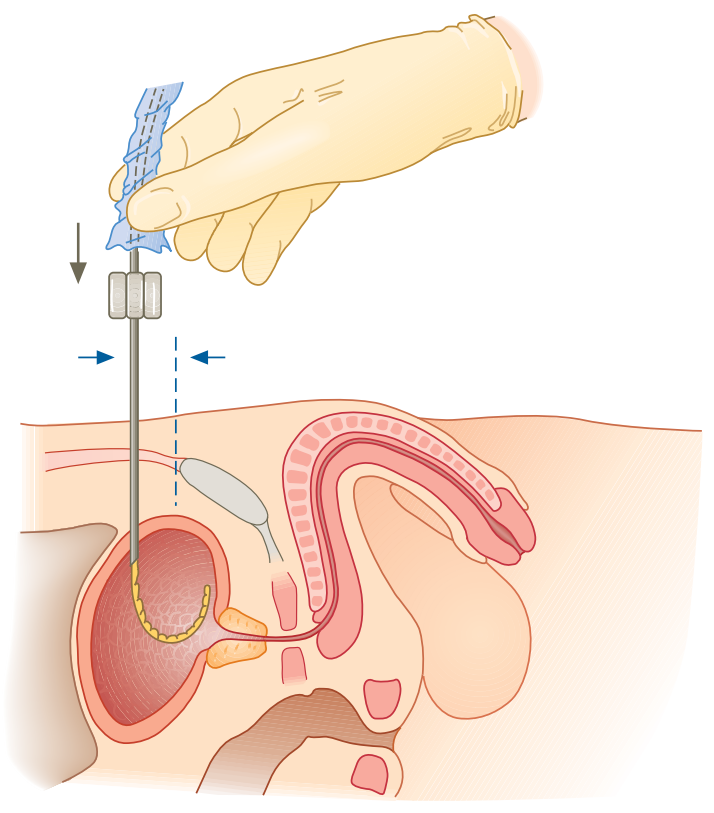

Punktion der Blase, Einführen des Katheters

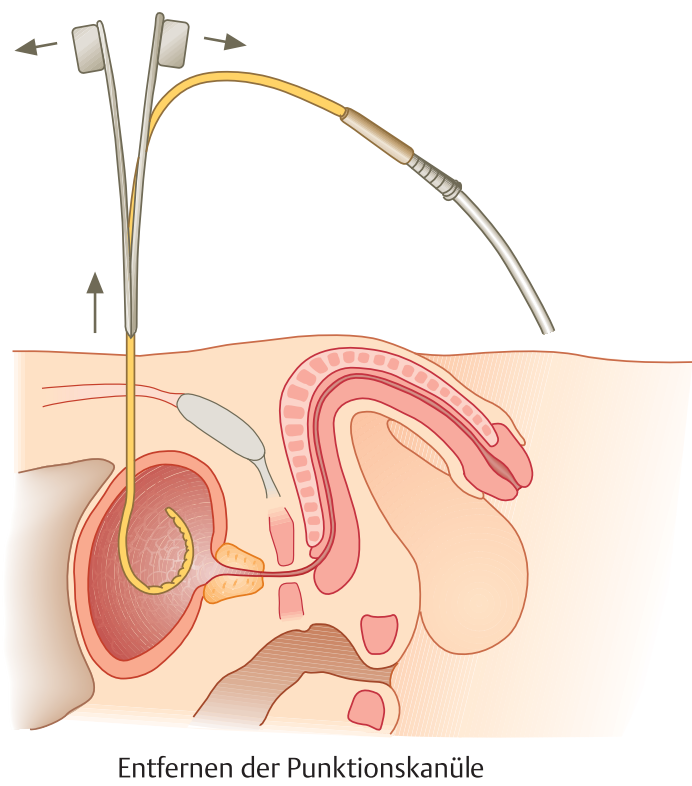

Abb. 14 Schematische Darstellung der Anlage einer suprapubischen Harnableitung (Quelle: Sökeland, Haßdenteufel, Krieger, Lutz. Urologie für Pflegeberufe. Thieme 2000).

Makrohämaturie ist die vermehrte Ausscheidung von Erythrozyten über den Urin, welcher dadurch erkennbar rot gefärbt ist. Die Makrohämaturie ist ein Symptom und kein Krankheitsbild.

Bei einer „falschen“ Makrohämaturie wird der Urin nicht durch eine Blutung, sondern durch Medikamente, Nahrungsmittel oder andere Erkrankungen (zum Beispiel Porphyrie) rot gefärbt.

Anamnese und körperliche Untersuchung. Neben der allgemeinen Anamnese sollten Vorerkrankungen, welche mit einer echten Makrohämaturie einhergehen können, und die Medikamenteneinnahme (Antikoagulanzien) erfragt werden. Ebenso sollte der Patient bezüglich der Dauer, dem Intervall, der Schmerzhaftigkeit, dem Ablauf und der Stärke der Makrohämaturie befragt werden. Besteht eine initiale Makrohämaturie, kann die Blutung im Bereich der Harnröhre oder des Blasenhalses vermutet werden, bei terminaler Makrohämaturie eher im Bereich der Harnblase oder der oberen Harnwege.

Bei schmerzloser Makrohämaturie besteht immer der Verdacht auf ein Malignom im Bereich des Urogenitaltrakts (Niere, Ureter, Blase, Prostata, Urethra). Die schmerzhafte Makrohämaturie ist häufig bedingt durch entzündliche Prozesse im Urogenitaltrakt oder eine Urolitihiasis, die Lokalisation der Schmerzen und deren Qualität (dauerhaft, kolikartig, krampfartig) kann anamnestisch Hinweise auf die mögliche Ursache wie zum Beispiel Steinkolik, akute Zystitis etc. geben.

Eine schmerzlose Makrohämaturie ist immer suspekt auf das Vorliegen eines Malignoms im Bereich des Urogenitaltrakts.

Bei der körperlichen Untersuchung müssen die wichtigsten Basisparameter (Puls, Blutdruck, Temperatur, Hautkolorit) zur Einschätzung der Kreislaufsituation des Patienten erhoben werden. Im Rahmen der kompletten urologischen Untersuchung sollten äußere Verletzungen im Bereich des Urogenitaltrakts ausgeschlossen werden. Falls möglich, sollte der Urin makroskopisch inspiziert werden, um die Stärke der Makrohämaturie einzuschätzen (dunkelrot, rosé, frischblutig, altblutig etc.).

Diagnostik. Eine Blutbilduntersuchung gibt Anhalt über das Ausmaß und die aktuelle Relevanz der Makrohämaturie, der Gerinnungsstatus liefert nützliche Zusatzinformationen [17,20-22].

Zeigt sich in der Notfalldiagnostik ein kreislaufstabiler Patient und ist die Notfallsituation beherrscht, muss bei jeder auch passager bestehenden Makrohämaturie die 
weitere abklärende Diagnostik (zum Beispiel Sonografie, Urethrozystoskopie, Ausscheidungsurogramm etc.) zum Ausschluss eines malignen Geschehens erfolgen.

Bei kreislaufstabilem Patienten und beherrschter Notfallsituation folgt die weitere abklärende Diagnostik zum Ausschluss eines malignen Geschehens.

Harnblasentamponade. Bei massiven Blutungen kann es zur Bildung und dem Abgang von Blutkoageln kommen und es kann eine Harnblasentamponade entstehen.

Bei einer Harnblasentamponade gerinnt das Blut in der Harnblase. Weder der Urinfluss noch die im Urin enthaltene Urokinase reichen bei massiven Blutungen aus, um eine Blasentamponade zu verhindern. Besteht eine Blasentamponade, ist eine suffiziente Miktion oft nicht mehr möglich und es bestehen massive Schmerzen.

Klinisch zeigt sich ein Zustand ähnlich dem akuten Harnverhalt: Schmerzen im Unterbauch und ein palpabler Unterbauchtumor. Möglicherweise berichtet der Patient über den urethralen Abgang von Blutkoageln. Bei einer massiven Blutung können ein deutlich reduzierter Allgemeinzustand und eine instabile Kreislaufsituation vorliegen.

Besteht eine Blasentamponade, ist eine suffiziente Miktion oft nicht mehr möglich und es bestehen massive Schmerzen.

Therapeutisch hat die Stabilisierung der Kreislaufverhältnisse und die rasche Freispülung der Harnblase und Entfernung der Bluttamponade absolute Priorität.

Nach der Stabilisierung der Kreislaufverhältnisse sollte ein möglichst großlumiger Spülkatheter transurethral eingelegt werden, über welchen manuell mithilfe einer Blasenspritze die Koagel ausgeräumt werden können. Gelingt dies und die Blase ist leer, können sich die Blasengefäße kontrahieren.
Zur Vermeidung der erneuten Bildung von Koageln bzw. einer Blasentamponade ist eine Spülung der Blase (zum Beispiel mit physiologischer Kochsalzlösung) unerlässlich. Da ein Spülkatheter und eine Blasenspülung im Notfall vor Ort meist nicht verfügbar sind, kann bis zum Transport in eine urologische Klinik/Einheit auch ein normaler transurethraler Dauerkatheter mit einem möglichst großen Lumen eingelegt werden. Auch über diesen kann versucht werden, Teile der Blasentamponade zu entfernen. Zusätzlich kann zum Beispiel eine Blutung aus dem Bereich des Blasenhalses (zum Beispiel durch Prostatavarizen) durch Zug am Katheter oder eine urethrale Blutung durch Katheterkompression verringert werden.

Kann eine Blasentamponade nicht via Katheter evakuiert werden oder persistiert die Blutung, ist eine rasche weitere diagnostische Abklärung und Lokalisation der Blutung mit konsekutiver Therapie auch in Narkose bei meist ausgeprägten Schmerzen zwingend notwendig [17,20-22].

Mechanische Manipulationen. Manipulationen (auch autoerotisch) mit Fremdkörpern im Bereich der Urethra und Harnblase können zu einem akuten Harnverhalt durch eine mechanische Verlegung und/oder eine Makrohämaturie durch Verletzung der Urethral- bzw. Blasenschleimhaut führen. Hier ist die Anamnese richtungsweisend. Bei noch in situ befindlichen Fremdkörpern ist die weiterführende Diagnostik in einer urologischen Klinik/Einheit und, wenn möglich, endoskopische Entfernung notwendig. Hier sollte im Notfall nicht vor Ort versucht werden, das „Corpus delicti“ zu entfernen, um dadurch bedingten möglichen Komplikationen vorzubeugen bzw. diese dann in einer urologischen Klinik adäquat beherrschen zu können.

Selten: Bei jungen Patienten/Patientinnen mit einem Harnverhalt und/oder Makrohämaturie sollte als Ursache auch an eine Folge von Manipulationen mit Fremdkörpern gedacht werden. 
Kasuistik 1

16-Jähriger mit Notfallvorstellung bei akutem Harnverhalt. Die weitere Abklärung erbrachte den sonografischen Verdacht auf einen Blasenstein (siehe Sonobild), welcher in diesem Alter eine Rarität darstellt. Intraoperativ zeigte sich kein Blasenstein, sondern eine vor mehreren Jahren transurethral eingeführte Kindergeburtstagskerze, bereits mit Verkalkungen (Abb. 15).
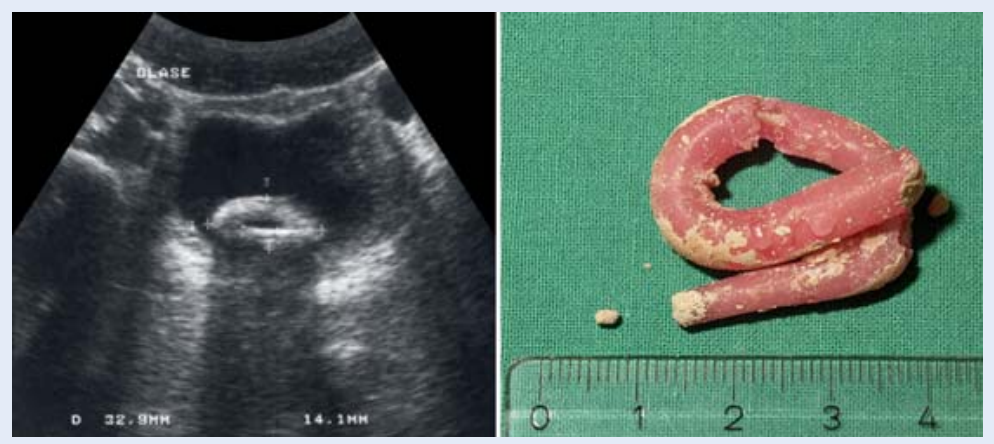

Abb. 15 Sonografisches Bild und intraoperativ geborgene Kindergeburtstagskerze mit Verkalkungen (Quelle: Axel Hegele).

\section{Kasuistik 2}

Wäscheleinendraht und Gummischlauch, welche in autoerotischer Absicht in die Harnblase eingeführt wurden, führten zu einem akuten Harnverhalt und mussten endoskopisch geborgen werden (Abb. 16). Das Ende der Wäscheleine war bei Eintreffen des Notarztes noch am Meatus urethrae externus sichtbar, konnte jedoch aufgrund der Verwicklung der Leine in der Harnblase nicht einfach vor Ort entfernt werden. Dies wurde vom
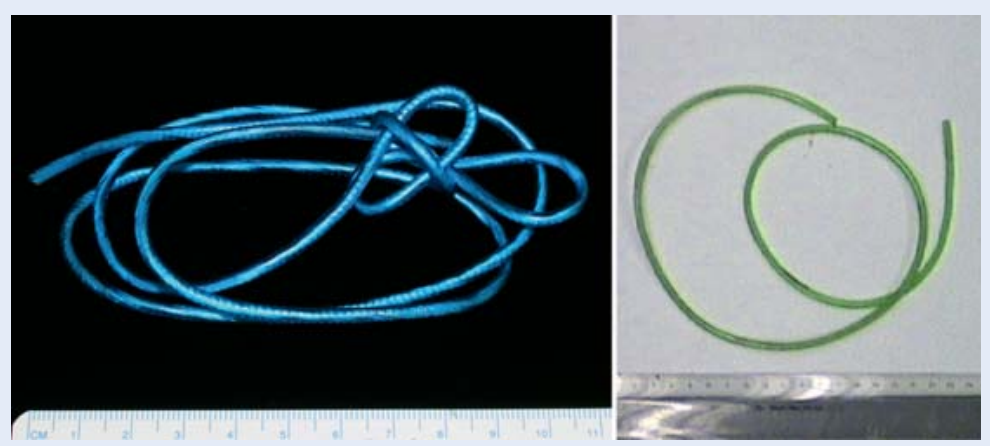

Abb. 16 Wäscheleinendraht und Gummischlauch, die in autoerotischer Absicht in die Harnblase eingeführt worden waren (Quelle: Axel Hegele).

Notarzt vor Ort auch nicht forciert, da er sich der Komplikationen eines „stärkeren Ziehens“ (wie Makrohämaturie, Harnröhrenverletzung etc.) bewusst war.

\section{Kasuistik 3}

Eine 21-jährige Patientin hatte gemeinsam mit dem Partner unter Drogeneinfluss autoerotische Manipulationen an der Harnröhre vorgenommen mit einer abschraubbaren Autodachantenne, die in der Blase „verschwand“. Notfallvorstellung mit Unterbauchschmerzen und Makrohämaturie. Abklärung mittels Röntgen des Unterbauchs. Die Antenne konnte transurethral-endoskopisch entfernt werden (Abb. 17).
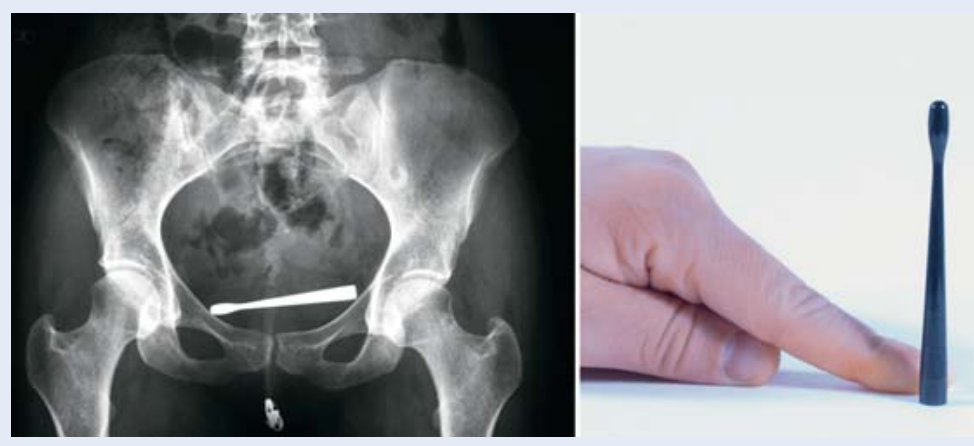

Abb. 17 Radiologischer Befund zu Kasuistik 3 und Corpus delicti nach transurethral-endoskopischer Entfernung (Quelle: Axel Hegele). 


\section{Kernaussagen}

Die vorausgegangenen Ausführungen zu den wichtigsten und häufigsten urologischen Notfallsituationen konnten zeigen, dass durch gezielte Anamnese, gründliche körperliche Untersuchung und einfache Labor- und Ultraschalluntersuchungen eine verhältnismäßig gute und zielsichere Diagnostik bereits am Ort der Erstversorgung möglich sind. Einige der Differenzialdiagnosen erfordern schon im Verdachtsfall eine unverzügliche Einweisung in eine Institution, in der eine weitere Abklärung und ggf. auch eine invasive oder operative Therapie möglich sind. Voraussetzung ist - wie so oft - dass man „daran denkt“ und die Organe des Urogenitaltrakts bei Kindern und Erwachsenen als relativ häufige Ursache einer akuten Erkrankung bzw. klinischen Notfallsituation in die differenzialdiagnostischen Überlegungen mit einbezieht.

\section{Über die Autoren}

Peter J. Olbert

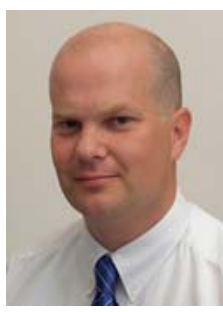

Jahrgang 1969. PD Dr. med. Studium der Humanmedizin in Regensburg und München (TU), 2 Jahre Weiterbildungszeit Allgemein- und Unfallchirurgie im Kreiskrankenhaus Freilassing, dort Erwerb der Fachkunde Rettungsdienst und 2-jährige Tätigkeit als Notarzt. Weiterbildung zum Facharzt für Urologie in München und Marburg. Seit 1999 in der Klinik für Urologie und Kinderurologie, Universitätsklinikum Marburg, dort seit 2004 Oberarzt und seit 2008 leitender Oberarzt und Koordinator des Prostatakarzinomzentrums. Habilitation 2009. Zusatzweiterbildung für Medikamentöse Tumortherapie seit 2008.

\section{Martin Ludwig}

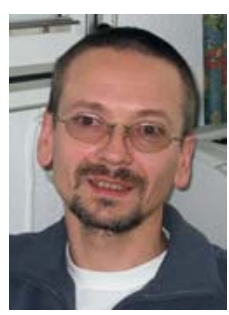

Jahrgang 1962. Dr. med. Studium der Humanmedizin in Marburg. Urologische Ausbildung an den Universitätskliniken Göttingen und Gießen, langjährig Leiter der Infektionssprechstunde der Klinik und Poliklinik für Urologie und Kinderurologie, Gießen. Mitglied im AK Infektiologie der Deutschen Urologen. Zusatzbezeichnungen Medikamentöse Tumortherapie und Andrologie, sowie Certified Andrologist der European Association of Andrology (EAA). Mitglied der Deutschen Gesellschaft für Ultraschall in der Medizin (DEGUM), Ausbilderstatus für die Sektion Urologie. Seit 2005 urologische Praxis Dres. v. Keitz, Schwickardi, Ludwig.

\section{Axel Hegele}

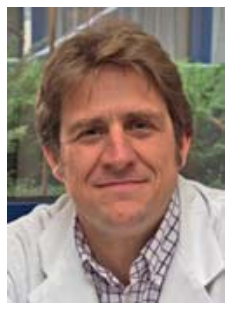

Jahrgang 1970. PD Dr. med. Studium der Humanmedizin (Approbation 1998) und Weiterbildung zum Facharzt an der Marburger Universitätsklinik (2005). Seit Januar 2006 Oberarzt in der Klinik für Urologie und Kinderurologie, Universitätsklinikum Marburg. Habilitation 2009. Zusatzweiterbildung Fachkunde Strahlenschutz und Medikamentöse Tumortherapie.

\section{Korrespondenzadresse}

PD Dr. med. Peter J. Olbert

Universitätsklinikum Gießen und Marburg

Standort Marburg

Baldingerstraße

35043 Marburg

Telefon: 06421/58-62513/62252

Fax: 06421/58-65590

E-Mail: peter.olbert@med.uni-marburg.de 


\section{Literatur}

1 Matthies C, Wagner W. Notfälle. In: Schmelz HU, Sparwasser C, Weidner W (Hrsg.). Facharztwissen Urologie. Differenzierte Diagnostik und Therapie. Heidelberg: Springer; 2010: 78-85

2 Ludwig M. Diagnosis and therapy of acute prostatitis, epididymitis and orchitis. Andrologia 2008; 40: 76-80

3 Chan PTK, Schlegel PN. Inflammatory conditions of the male excurrent ductal system. Part I. J Androl 2002; 23: 453-460

4 Pilatz A, Wagenlehner F, Hossain H, Weidner W. Acute Epididymitis: Etiology and inflammatory Parameters in a prospective Study. EAU Abstract 2010

5 Djakovic N, Plas E, Martínez-Piñeiro L et al. Urological Trauma. EAU guidelines online 2009: www.uroweb.org/gls/pdf/ Urological\%20Trauma\%202010.pdf

6 Waldert M, Klatte T, Schmidbauer J et al. Color Doppler sonography reliably identifies testicular torsion in boys. Urology 2010; 75: $1170-1174$

7 Grabe M, Bjerklund-Johansen TE, Botto $\mathrm{H}$ et al. Guidelines on urological infections. EAU Guidelines online 2010. www.uroweb.org/ gls/pdf/Urological\%20Infections\%202010.pdf

8 Enders M, Turnwald-Maschler A, Regnath T. Antimicrobial resistance of Neisseria gonorrhoeae isolates from the Stuttgart and Heidelberg areas of Southern Germany. Eur J Clin Microbiol Infect Dis 2006; 25: 318-322

9 Roche-Nagle G, Rubin BB. Considerations in the diagnosis and therapy for acute loin pain. Am J Emerg Med 2009; 27: 254.e3-4

10 Gaspari R, Horst K. Emergency ultrasound and urinalysis in the evaluation of flank pain. Acad Emerg Med 2005; 12: 1180-1184

11 Grabe M, Bishop MC, Bjerklund-Johansen TE et al. Guidelines on urological Infections. In: EAU Guidelines 2010 Edition. Arnhem: EAU-Office; 2010: $63 \mathrm{ff}$.
12 Perminger GM, Tiselius HG, Assimos DG et al. 2007 Guideline for the management of ureteral calculi. Eur Urol 2007; 178: 2418 2434

13 Emberton M, Fitzpatrick JM. The Reten-World survey of the management of acute urinary retention: preliminary results. BJU Int 2008; 101: 27-32

14 Thomas K, Chow K, Kirby RS. Acute urinary retention: a review of the aetiology and management. Prostate cancer and prostatic diseases 2003; 7: $32-37$

15 Cathcart P, van der Meulen J, Armitage J et al. Incidence of primary and recurrent acute urinary retention between 1998 and 2003 in England. J Urol 2006; 176: 200-204

16 Jacobsen SL, Jacobsen DJ, Girman CJ et al. Natural history of prostatism: risk factors for acute urinary retention. J Urol 1997; 158: $481-487$

17 Otto U, Göthe E. Urologische Notfallsituationen. In: Hautmann (Hrsg.). Urologie, 4. Aufl. Heidelberg, Berlin: Springer, 2010

18 Rosenstein D, McAninch JW. Urologic emergencies. Med Clin North Am 2004; 88: 495-518

19 Vilke GM, Ufberg JW, Harrigan RA et al. Evaluation and treatment of acute urinary retention. J Emerg Med 2008; 35: 193-198

20 Hicks D, Li CY. Management of macroscopic haematuria in the emergency department. Emerg Med J 2007; 24: 385-390

21 Sokolosky MC. Hematuria. Emerg Med Clin North Am 2001; 19: 621-632

22 Hay AD, Hamilton W, Sharp D et al. Macroscopic haematuria and urological cancer. Br J Gen Pract 2003; 53: 241-242 


\section{CME-Fragen}

\section{CME.thieme.de}

\section{CME-Teilnahme}

- Viel Erfolg bei lhrer CME-Teilnahme unter http://cme.thieme.de

- Diese Fortbildungseinheit ist 12 Monate online für eine CME-Teilnahme verfügbar.

- Sollten Sie Fragen zur Online-Teilnahme haben, unter http://cme.thieme.de/hilfe finden Sie eine ausführliche Anleitung.

\section{1}

Welcher Keim ist ein typischer Auslöser der akuten Epididymitis beim jungen Mann?
A Staphylococcus aureus
B Streptococcus spp.
C Chlamydia trachomatis
D Pseudomonas aeruginosa
E Herpes-simplex-Virus Typ II

2

Was versteht man unter dem Prehn'schen Zeichen in der klinischen Diagnostik der Hodentorsion?
A Schmerz verstärkt sich beim Drehen des Hodens nach lateral.
B Schmerz verstärkt sich beim Drehen des Hodens nach medial.
C Schmerz lässt beim Anheben des Hodens nach.
D Schmerz bessert sich bei Applikation von Wärme.
E Schmerz lässt beim Anheben des Hodens nicht nach.

3

Arterielle Flusssignale im FKDS des Hodens ...
A ... schließen eine Hodentorsion sicher aus.
B ... können trotz bestehender Hodentorsion vorhanden sein.
C ... sind ohne Aussagekraft, da eine zuverlässige Doppler-Sonografie des Hodens wegen des kleinen Gefäßkalibers sowieso nicht möglich ist.
D ... sind ein sicherer Nachweis einer Hodentorsion.
E ... sind bei einer akuten hämorrhagischen Infarzierung des Hodens nie nachweisbar.

\section{4}

Welches ist kein häufiges Begleitsymptom der Nierenkolik?
A Übelkeit
B Erbrechen
C Meteorismus
D Subileus
E Ikterus

\section{5}

Welche ist die richtige Maßnahme bei Harnstauungsniere mit Flankenschmerzen und hohem Fieber?
A sofortige Entlastung der Niere
B Wärmeapplikation
C Bewegung und reichlich Flüssigkeitszufuhr
D Asservierung eines 24-h-Sammelurins
E antipyretische Medikation 


\section{CME-Fragen}

Urologische Notfälle: Klinische Präsentation, Diagnostik und Therapie

\section{6}

Der Kolikschmerz beim distalen

Ureterstein strahlt häufig aus ...
A $\quad$... in das Gesäß.
B ... in den Oberbauch.
C ... zwischen die Schulterblätter.
D ... ins äußere Genitale und die Oberschenkelinnenseite.
E ... in den ipsilateralen Arm.

\section{7}

Was ist keine typische infravesikale Ursache für einen Harnverhalt?
A Prostatavergrößerung
B Harnröhrenstriktur
C Blasensteine
D narbige Phimose
E Harnröhrenverletzung

8

Welches Krankheitsbild ist nicht
A Nierentumor
B Harnblasenkarzinom
C Harnsteine
D Harnwegsinfekt
E Porphyrie

9

Welches ist die Untersuchung der Wahl zum Ausschluss eines Harnblasentumors bei schmerzloser Makrohämaturie?
A Sonografie
B Computertomografie
C Urethrozystoskopie
D Kernspintomografie
E Tumormarkerbestimmung

10

Die häufigste Ursache für eine akute Obstruktion des oberen Harntrakts ist/sind ...
A ... ein Harnleiterstein.
B ... Tumorkompression von außen.
C ... radiogene Strikturen.
D ... ein Ureterabriss nach Dezelerationstrauma.
E ... primäre Uretertumoren. 